Minireview

\title{
Genetic and genomic insights into age at natural menopause
}

\author{
Francisco Jesus Moron, Agustin Ruiz and Jose Jorge Galan
}

Address: Department of Structural Genomics, Neocodex SL, Avda, Charles Darwin 6, Acceso A, Isla de la Cartuja, 41092, Sevilla, Spain.

Correspondence: Jose Jorge Galan. Email: jjgalan@neocodex.es

\begin{abstract}
The age at natural menopause shows great variability. It has been proposed that early age at menopause is a risk factor for osteoporosis and cardiovascular disease, whereas later age at menopause is a risk factor for breast cancer. In addition, it is thought that the genetic factors accounting for the genetic variability in age at menopause could also play a role in those diseases, as well as infertility in women. In this minireview we comment on the latest genetics and genomics insights into age at natural menopause.
\end{abstract}

\section{Why study the genetic factors influencing the age at natural menopause?}

Natural menopause is defined as the cessation of menstrual cycles for 12 consecutive months, resulting from a lack of ovarian follicular activity. It is a biological process occurring between approximately 40 and 60 years of age, with a mean value at approximately 51 years [1].

In recent decades, the timing of menopause has gained attention for several reasons. Firstly, in most Western countries, a trend towards increased maternal age has been recognized. In this context, prediction of an early menopause (that is, early onset of subfertility or infertility) is of clinical significance [2]. Secondly, early onset of menopause is associated with a higher risk of cardiovascular diseases and osteoporosis, whereas a later menopausal age has been associated with an increased risk of breast cancer [1]. In addition, human population studies have reported that older age at menopause is positively correlated with longevity [3]. Therefore, it is hypothesized that those factors influencing age at natural menopause (ANM) may also play a role in the etiology and/or course of those common diseases, in addition to an association with infertility and aging.

Although some environmental and lifestyle factors influencing ANM have been identified [1], a great heritability for ANM has been estimated, ranging from $31 \%$ to $53 \%$ [4], and $70 \%$ to $85 \%$ in singleton sisters [2]. Thus, it is assumed that an important genetic component also accounts for the variability observed in the timing of menopause. In this minireview we will comment on the latest insights into ANM.

\section{Genetics and genomics of age at natural menopause}

The search for genetic variants influencing ANM has mainly focused on candidate molecular pathways for which previous clinical and/or biological evidence suggested an involvement in ovary senescence.

For instance, given the pivotal role of estrogens in female reproduction, some genes participating in the synthesis, mechanism of action and degradation of those hormones have been analyzed and proposed as predictor factors for ANM, such as ESR1 (estrogen receptor alpha) gene [5], CYP17A1 (cytochrome P450, family 17, subfamily A, polypeptide 1) gene [6] or CYP1B1 (cytochrome P450, family 1 , subfamily $B$, polypeptide 1) gene [7]. Other studies have analyzed genes with a role in thrombophilia and vascular homeostasis, since ovarian senescence is partly due to aging of the vascular supply to the ovary. In this context, coagulation factors V and VII have been associated with reduced and increased ANM, respectively [8,9]. However, other studies could not replicate some of these findings, and the real effect of these variants on ANM remains to be clarified.

Other researchers have developed hypothesis-free strategies that do not focus on candidate molecular pathways. Instead, these strategies interrogate thousands of genetic markers, spread along the genome, to identify genomic loci that could be involved in the trait under study, without prior assumptions about the underlying mechanism. Two of those strategies have been applied to the study of ANM: linkage studies and genome-wide association studies (GWASs).

van Asselt et al. [10] reported a genome-wide linkage analysis in extremely concordant and discordant sibling pairs. One year later, Murabito et al. [11] carried out a genome-wide linkage analysis of ANM in a populationbased sample. The linkage signals detected in both studies were different, and thus could not be replicated. The

ANM = age at natural menopause; $\mathrm{CEPH}=$ Centre d'Etude du Polymorphisme Humain; GWAS = genome-wide association study; SNP = single nucleotide polymorphism. 
Table 1

\begin{tabular}{|c|c|c|c|c|c|c|c|c|}
\hline $\begin{array}{l}\text { Chromosomal } \\
\text { region }\end{array}$ & SNP & Position & $\begin{array}{l}\text { Minor } \\
\text { allele }\end{array}$ & MAF & beta & $\begin{array}{l}\text { Standard } \\
\text { error }\end{array}$ & $P$-value & Reference \\
\hline \multirow[t]{10}{*}{$19 q 13.4$} & rs12611091 & 60492141 & C & 0.48 & 0.33 & 0.053 & $6.6 \times 10^{-10}$ & [13] \\
\hline & rs1551562 & 60506693 & G & 0.25 & -0.428 & 0.070 & $1.04 \times 10^{-9}$ & [12] \\
\hline & & & & 0.23 & -0.43 & 0.062 & $2.6 \times 10^{-12}$ & [13] \\
\hline & rs1172822 & 60511657 & $\mathrm{~T}$ & 0.39 & -0.391 & 0.060 & $6.28 \times 10^{-11}$ & [12] \\
\hline & & & & 0.37 & -0.49 & 0.054 & $1.8 \times 10^{-19}$ & [13] \\
\hline & rs7246479 & 60516144 & $\mathrm{~T}$ & 0.48 & 0.36 & 0.052 & $2.3 \times 10^{-12}$ & [13] \\
\hline & rs2384687 & 60523000 & $\mathrm{C}$ & 0.40 & -0.381 & 0.059 & $1.39 \times 10^{-10}$ & [12] \\
\hline & & & & 0.39 & -0.47 & 0.053 & $2.4 \times 10^{-18}$ & [13] \\
\hline & rs897798 & 60525566 & G & 0.48 & -0.308 & 0.056 & $3.91 \times 10^{-8}$ & [12] \\
\hline & & & & 0.47 & -0.40 & 0.052 & $1.1 \times 10^{-14}$ & [13] \\
\hline \multirow[t]{2}{*}{$20 q 12.3$} & rs236114 & 5883385 & $A$ & 0.21 & 0.495 & 0.077 & $9.71 \times 10^{-11}$ & [12] \\
\hline & rs16991615 & 5896227 & A & 0.058 & 1.07 & 0.11 & $1.2 \times 10^{-21}$ & [13] \\
\hline
\end{tabular}

MAF, minor allele frequency; SNP, single nucleotide polymorphism.

different study designs in the two reports are likely, at least partially, to account for these discrepancies. Nonetheless, both studies proposed attractive candidate loci for age at menopause, such as the BCL2 (B-cell CLL/lymphoma 2) gene on 9q21.3 due to its role in apoptosis [10], or the GNRH1 (gonadotropin-releasing hormone 1 (luteinizingreleasing hormone)) gene close to $8 \mathrm{p} 22$, which is a key molecule in the hypothalamic-pituitary-gonadal axis that controls human reproduction [11].

Two recent articles report the first genome-wide analyses on ANM. Stolk et al. [12] carried out a two-stage GWAS. The first stage combined 2,368 samples from the Rotterdam Study with 611 samples from the TwinsUK Study. The second stage comprised 2,560 samples of four additional cohorts of postmenopausal females of European ancestry. The authors identified six common single nucleotide polymorphisms (SNPs) that were significant genome-wide in the combined stages 1 and 2 of their study. Four SNPs were located in the same haplotype block within the chromosomal region 19q13.4; rs236114 was located on 20p12.3, and rs7333181 on 13934 [12]. Stolk and colleagues reported a joint analysis of two GWASs from the Nurses' Health Study $(n=2,287)$ and the Women's Genome Health Study $(n=15,151)$.They found 13 markers showing strong association in the joint analysis. These markers were clustered in four different chromosomal regions (5q35.2, 6p24.2, 19q13.42 and 20p12.3) [13]. Interestingly, two of those regions, 19q13.4 and 20p12.3, were identified in both independent studies (Table 1).
The effect sizes for four markers at 19q13.4 are virtually the same in both studies. Two markers (rs12611091 and rs7246479) at this region were detected as significant signals only in the study by He et al. [13]. It is possible that two markers were not genotyped or they could have failed quality criteria in the study by Stolk et al. [12]. Interestingly, these two markers seem to be associated with a later age at menopause (beta $>0$ ), the opposite effect to that observed for the other significant markers at the same region. It is possible that minor alleles of rs12611091 and rs7246479 are in 'trans' with the minor alleles of the other four markers at the same haplotypic block.

The two significant markers at region 20q12.3 show different effect sizes (Table 1). According to HapMap data (phase III, CEU analysis panel comprising 30 trios of US residents with ancestry from Northern and Western Europe, collected by the Centre d'Etude du Polymorphisme Humain (CEPH)), both markers are in complete linkage disequilibrium $\left(D^{\prime}=1\right)$. Therefore, they could be proxies for the same causative genetic variant. As rs16991615 shows a larger effect size, it is tempting to speculate that rs16991615 is closer to a causal variant than rs236114, which would account for the observed differences in the effect sizes between both markers.

\section{Lessons learned from new insights into age of menopause}

These two recent GWASs have highlighted the potential implications of new loci in the timing of natural menopause. Little is known about the function of these new candidate genes. $\mathrm{HK}_{3}$ (hexokinase 3 (white cell)) is 
involved in carbohydrate metabolism, and UIMC1 (ubiquitin interaction motif containing 1) can act as a transcriptional repressor and is involved in DNA damage resistance, G2/M checkpoint control, and DNA repair through binding with $B R C A 1$ (breast cancer 1 early onset) gene complexes. SYCP2L (synaptonemal complex protein 2-like) knockout mice are sterile in male and subfertile with sharply reduced litter size in female mice. The BRSK1 (BR serine/threonine kinase 1) gene is required for neuronal polarization, while the MCM8 (minichromosome maintenance complex component 8) gene is involved in DNA replication [12,13]. Therefore, these genes and others involved in the same molecular pathways have become very attractive candidates for further investigations in ANM.

In addition, it should be borne in mind that the significant SNPs reported in both studies may not be the causal variants, but they could be in linkage disequilibrium with the causal variants. In this sense, other loci close to or within the haplotype blocks reported in both studies, such as the genes TRMT6 (tRNA methyltransferase 6 homolog (S. cerevisiae)), SUV42OH2 (suppressor of variegation 4-20 homolog 2 (Drosophila)), ZNF346 (zinc finger protein 346), HSPBP1 (HSPA (heat shock 7okDa) binding protein, cytoplasmic cochaperone 1), GCM2 (glial cells missing homolog 2 (Drosophila)) or UNC5A (unc-5 homolog A (C. elegans)), could be related to ANM and they should also be analyzed in further studies.

Beyond the successful identification of two strong, consistent and novel loci for ANM, further re-analyses of datasets produced during both projects may have a deep impact on the validation or refutation of previously proposed candidate genes for ANM [5-9].

Moreover, the application of novel analytical techniques, such as epistasis or pathway-based approaches to available datasets, may add new layers of knowledge and will produce novel discoveries by recycling generated genotypes. Thus, we anticipate that the results obtained in both GWASs might represent only the tip of an iceberg of ongoing research.

\section{How this novel knowledge can be used to potentially treat infertility in young women}

The latest insights into ANM have no immediate medical applications in young women. To date, there is no complete model of causation able to predict, with some accuracy, the timing of menopause in women.

In fact, the markers proposed by Stolk et al. and He et al. account for only $1.1 \%$ and $2.69 \%$, respectively, of the variability observed in the samples analyzed [12,13], which shows that there must be additional environmental and genetic factors accounting for the observed variability. The identification of those factors is necessary to build a predictive model for ANM. This model will allow the early recognition of women at risk of early menopause, and hence a shorter reproductive life span, as well as the application of preventive measures, from the initial stages of life, to women at risk.

Finally, the identification of genetic variants influencing ANM has potential implications for the treatment of infertile women. Those genes associated with ANM could be used as targets to develop new drugs aimed at delaying the end of the reproductive capacity in women by, for instance, preventing premature depletion of the ovarian pool of oocytes.

\section{Competing interests}

FJM and JJG are employees of Neocodex SL. AR is a stakeholder of Neocodex SL. In addition, the authors declare that they have no non-financial competing interests.

\section{Authors' contributions}

FJM and JJG made substantial contributions to conception and design, acquisition of data and analysis and interpretation of data. AR and JJG were involved in drafting the manuscript or revising it critically for important intellectual content. FJM, AR and JJG have given final approval of the version to be published.

\section{Acknowledgements}

We are deeply grateful to Antonio Gonzalez for his critical revision of the manuscript. This work has been partially supported by the Ministerio de educación y Ciencia of Spain (CIT-090100-2007-57) and the Corporación Tecnológica de Andalucía (07/126).

\section{References}

1. Kok HS, van Asselt KM, van der Schouw YT, Peeters PH, Wijmenga C: Genetic studies to identify genes underlying menopausal age. Hum Reprod Update 2005, 11:483-493.

2. de Bruin JP, Bovenhuis $H$, van Noord PA, Pearson PL, van Arendonk JA, te Velde ER, Kuurman WW, Dorland M: The role of genetic factors in age at natural menopause. Hum Reprod 2001, 16:2014-2018.

3. Hanna CW, Bretherick KL, Gair JL, Fluker MR, Stephenson MD, Robinson WP: Telomere length and reproductive aging. Hum Reprod 2009, 24:1206-1211.

4. Snieder H, MacGregor AJ, Spector TD: Genes control the cessation of a woman's reproductive life: a twin study of hysterectomy and age at menopause. J Clin Endocrinol Metab 1998, 83:1875-1880.

5. Weel AE, Uitterlinden AG, Westendorp IC, Burger $H$, Schuit SC, Hofman A, Helmerhorst TJ, van Leeuwen JP, Pols HA: Estrogen receptor polymorphism predicts the onset of natural and surgical menopause. $J$ Clin Endocrinol Metab 1999, 84:3146-3150.

6. Hefler LA, Grimm C, Heinze G, Schneeberger C, Mueller MW, Muendlein A, Huber JC, Leodolter S, Tempfer CB: Estrogenmetabolizing gene polymorphisms and age at natural menopause in Caucasian women. Hum Reprod 2005, 20: 1422-1427.

7. Long JR, Shu XO, Cai Q, Wen W, Kataoka N, Gao YT, Zheng W: CYP19A1 genetic polymorphisms may be associated with obesity-related phenotypes in Chinese women. Int $J$ Obes (Lond) 2007, 31:418-423. 
8. van Disseldorp J, Broekmans FJ, Peeters PH, Fauser BC, van der Schouw YT: The association between vascular functionrelated genes and age at natural menopause. Menopause 2008, 15:511-516.

9. Tempfer CB, Riener EK, Keck C, Grimm C, Heinze G, Huber JC, Gitsch G, Hefler LA: Polymorphisms associated with thrombophilia and vascular homeostasis and the timing of menarche and menopause in $\mathbf{7 2 8}$ white women. Menopause 2005, 12:325-330.

10. van Asselt KM, Kok HS, Putter $\mathrm{H}$, Wijmenga $\mathrm{C}$, Peeters $\mathrm{PH}$, van der Schouw YT, Grobbee DE, te Velde ER, Mosselman S, Pearson PL: Linkage analysis of extremely discordant and concordant sibling pairs identifies quantitative trait loci influencing variation in human menopausal age. Am J Hum Genet 2004, 74:444-453.

11. Murabito JM, Yang Q, Fox CS, Cupples LA: Genome-wide linkage analysis to age at natural menopause in a community-based sample: the Framingham Heart Study. Fertil Steril 2005, 84:1674-1679.
12. Stolk L, Zhai G, van Meurs JB, Verbiest MM, Visser JA, Estrada K, Rivadeneira F, Williams FM, Cherkas L, Deloukas $P$, Soranzo N, de Keyzer JJ, Pop VJ, Lips P, Lebrun CE, van der Schouw YT, Grobbee DE, Witteman J, Hofman A, Pols HA, Laven JS, Spector TD, Uitterlinden AG: Loci at chromosomes 13, 19 and 20 influence age at natural menopause. Nat Genet 2009, 41:645-647.

13. He C, Kraft P, Chen C, Buring JE, Paré G, Hankinson SE, Chanock SJ, Ridker PM, Hunter DJ, Chasman DI: Genomewide association studies identify loci associated with age at menarche and age at natural menopause. Nat Genet 2009, 41:724-728.

Published: 06 August 2009

doi:10.1186/gm76

(C) 2009 BioMed Central Ltd 